\title{
Outcome after In-Hospital Rebleeding of Rupture of Intracranial Aneurysms
}

\author{
Jenny Kienzler ${ }^{1}$ Serge Marbacher ${ }^{1}$ Luca Remonda ${ }^{2}$ Jehuda Soleman ${ }^{1}$ Janine Ai Schlaeppi ${ }^{1}$ \\ Ulrich Leupold ${ }^{1}$ Javier Fandino ${ }^{1}$
}

${ }^{1}$ Department of Neurosurgery, Kantonsspital Aarau, Aarau, Switzerland

2 Division of Neuroradiology, Department of Radiology, Kantonsspital Aarau, Aarau, Switzerland

Address for correspondence Jenny Kienzler, MD, c/o Editorial Office, Department of Neurosurgery, Kantonsspital Aarau, Tellstrasse, 5000 Aarau, Switzerland (e-mail: neurosurgery@ksa.ch; jenny.kienzler@ksa.ch).

J Neurol Surg A 2016;77:207-221.

\begin{abstract}
Background After initial subarachnoidal hemorrhage (SAH), due to an intracranial aneurysm, rebleeding is known as a factor influencing the devastating outcome. This complication has been reported to occur in $\sim 4 \%$ of the patients admitted with aneurysmal SAH. Moreover, ultra-early rebleeding within the first 24 hours might occur in 9 to $17 \%$ of the cases ( $40-87 \%$ appearing in the first 6 hours). Risk factors influencing this situation include increasing aneurysm size, deterioration of neurologic deficits, angiography within 3 hours of bleeding, sentinel symptoms, and the loss of consciousness at initial bleeding. The aim of this retrospective study was to assess factors and potential risk factors related to rebleeding, specifically the interval from initial SAH to rebleeding.

Material and Methods From a consecutive series of 243 patients who experienced aneurysmal SAH, we identified 28 patients (11.5\%; 12 men, 16 women; mean age: $58 \pm 10$ years) who developed in-hospital rebleeding during this 49-month study (2009-2013). Demographic, radiologic, and clinical characteristics including hemodynamic parameters were analyzed.

Results Rebleeding was fatal in 20 of the 28 patients (71\%) and caused severe neurologic deficits (Glasgow Outcome Scale: 3; modified Rankin Scale: 5 ) in 3 (29\%) of the remaining 8 survivors. Rebleeding occurred within the first 4 hours in 15 patients (54\%) within 7, 24, and 48 hours in 17 (61\%), 6 (21\%), and 1 (4\%) patient, respectively. In this series, the medium arterial blood pressure was $98 \pm 11 \mathrm{~mm} \mathrm{Hg}$ at arrival at the emergency department, $88 \pm 10 \mathrm{~mm} \mathrm{Hg}$ before rebleeding, and it dramatically increased to $124 \pm 22 \mathrm{~mm} \mathrm{Hg}$ at rebleeding. For the patients with rebleeding after aneurysmal SAH, initial sentinel headache $(79 \%)$ and loss of consciousness $(68 \%)$ were

Keywords

- subarachnoid hemorrhage

- rebleeding

- risk factor

- intracranial aneurysm

- outcome the common presenting symptoms. The World Federation of Neurological Societies grade was documented on admission as follows: $1-3(n=14$ [50\%]); $4-5$ ( $n=14$ [50\%]). A Fisher grade 4 was documented in $82 \%$ of the cases on the initial computed tomography (CT) scan. Overall, $42 \%$ of the cases underwent endovascular $(n=6)$ or microsurgical occlusion of the aneurysm $(n=6)$. The rest of the patients $(n=16,58 \%)$ did not underwent occlusion of the aneurysm because of poor clinical status. Digital substraction angiography was performed in $61 \%$ of the cases.
\end{abstract}

received

October 18, 2014

accepted after revision

September 30, 2015

published online

January 25, 2016 (c) 2016 Georg Thieme Verlag KG

Stuttgart · New York
DOI http://dx.doi.org/ 10.1055/s-0035-1570007. ISSN 2193-6315. 
Conclusion Possible factors increasing the risk of in-hospital rebleeding after aneurysmal SAH are high systolic blood pressure, sentinel headache, initial loss of consciousness, poor Hunt and Hess grade, high Fisher grade on initial CT, large aneurysm size, and the performance of angiography. Most of the rebleedings in patients in our center are likely to occur within 7 hours after admission. Based on our findings, we suggest that mobilization of the patient and maneuvers including invasive procedures should be restricted to a minimum during intensive care unit treatment prior to the occlusion of the ruptured aneurysm. Stabilization of blood pressure with adequate sedation and analgesia prior to occlusion can be considered preventive strategies against rebleeding.

\section{Introduction}

The major causes of morbidity and mortality of subarachnoidal hemorrhage $(\mathrm{SAH})$, caused by an intracranial aneurysm, are initial hemorrhage, rebleeding, and vasospasm with consecutive ischemia. ${ }^{1}$ Rebleeding is a devastating complication, associated with high mortality (80\%) and a poor outcome. ${ }^{2}$ It has been reported in earlier studies to occur in $4 \%$ of the patients admitted with $\mathrm{SAH}^{3}$ In current summaries of the literature in retrospective studies, the percentage found lies between $5 \%$ and $11 \%{ }^{1}$ Ultra-early rebleeding within the first 24 hours might occur in 9 to $17 \%$ of the cases, 40 to $87 \%$ appearing in the first 6 hours. ${ }^{4-6}$ More recent studies have found that the incidence of rebleeding might be underestimated due to ultra-early rebleeding during transfer or prior to initial imaging. ${ }^{4-7}$

A high rate of rebleeding is associated with untreated aneurysm, with delay in the treatment, and with the occlusion of the ruptured aneurysm. ${ }^{7-9}$ Therefore, it is now recognized that early intervention can effectively prevent rebleeding. ${ }^{10}$ And while so far rebleeding events often happened prior to the admission to medical care and patients died during or even before transport, the heightened awareness of the population as well as the optimized (shortened) rescue procedures now give us the chance to save those patients from a fatal outcome.

Potential risk factors associated with rebleeding have been identified: increasing aneurysm size, deterioration of neurologic deficits (Hunt and Hess [H\&H] grades 3 and 4), performance of angiography within 3 hours of bleeding, sentinel symptoms, and the loss of consciousness at initial bleeding. ${ }^{411-15}$ Our retrospective study aimed at assessing those risk factors. Additionally, demographic and clinical characteristics are presented.

\section{Materials and Methods}

\section{Patient Population}

This study included 243 patients who presented with acute aneurysmal SAH and were admitted to our institution with $\mathrm{SAH}$, confirmed by computed tomography (CT) scan or lumbar puncture during a 4-year period from November 2009 until December 2013.

During this 49-month study period, the mean overall mortality was $9.4 \%$ including cases with negative angiograph- ic findings. From these initial 243 patients, 28 (11.5\%) developed in-hospital SAH rebleeding (November 2009 to December 2013). Demographic and clinical findings were collected at admission and throughout the treatment with approval of the Kantonsspital Aarau institutional review board. Additionally, hemodynamic parameters at admission and during rebleeding and angiographic and CT findings, including Fisher grade, ${ }^{16}$ were documented. Analysis of radiologic findings included aneurysm characteristics (e.g., size, location).

The clinical status of the patients was defined by standard classifications such as Glasgow Coma Scale (GCS) score, WFNS (World Federation of Neurological Surgeons) grade, ${ }^{17}$ and H\&H grade. ${ }^{18}$ The inclusion criteria in this study were rebleeding (1) from ruptured saccular aneurysm and (2) after $\mathrm{SAH}$, which was confirmed by the increased volume of SAH on the CT scan. The outcome was assessed according to the Glasgow Outcome Scale (GOS) and the modified Rankin Score $(\mathrm{mRS}){ }^{19}$ In all cases the diagnosis of SAH was based on the initial noncontrast CT scan obtained within 1 hour after arrival at the emergency department. The severity was classified according to the Fisher scale (grade 1: no evidence of hemorrhage; grade 2: SAH with thickness $1 \mathrm{~mm}$; grade 3: $\mathrm{SAH}$ with thickness $>1 \mathrm{~mm}$; and grade 4 : intracerebral or intraventricular clot with diffuse or no SAH). ${ }^{16}$

Ruptures of the aneurysm were confirmed by CT angiography (CTA), magnetic resonance angiography (MRA), or digital substraction angiography (DSA). In the presence of an unsatisfactory definition of the aneurysm or adjacent vasculature in the CTA, a complementary DSA examination was performed.

All patients were admitted to the intensive care unit (ICU) to ensure optimal oxygenation and adequate hemodynamic management. ${ }^{20,21}$ Hemodynamic parameters, intracranial pressure (ICP), and cerebral perfusion pressure (CPP) were documented every hour. All patients underwent continuous monitoring of blood pressure values at admission and throughout the ICU treatment and transfer to the operating room and CT or angiography suites. In our institution, the upper limit of mean arterial pressure (MAP) for nontreated aneurysms is 75 to $105 \mathrm{~mm} \mathrm{Hg}$. To obtain the most accurate MAP and systolic blood pressure (SBP) "before rebleeding," values were documented immediately before clinical suspicion or ICP increase in the ICU, prior to transfer to an 
emergency CT scan. Fluid control was documented hourly and summarized twice a day. Initial routine laboratory examination included coagulation status, electrolytes, hematology, liver and renal function, and arterial blood gases.

Assessment of Rebleeding, Treatment, and Follow-Up After undergoing an emergency CT scan at the primary or referral hospital, patients admitted to our institution underwent another CT scan to obtain a standardized CTA. If the patient's condition was stable, DSA with three-dimensional reconstruction of the aneurysm was performed as part of the initial evaluation, especially in those with negative findings on CTA. For patients transferred directly to surgery, intraoperative DSA was performed before craniotomy for microsurgical occlusion of the aneurysm in our hybrid operating room. All patients who had surgical treatment underwent DSA and CT scanning immediately after the procedure within the operating room. ${ }^{22}$

The advantages of a hybrid operating room in terms of $\mathrm{SAH}$ rebleeding are that we do not have to wait for an appointment for angiography in the neuroradiologic department. Often this is time consuming, due to the limited capacity of the anesthesia. Moreover, additional transport and rearrangement of the patient can be avoided. After the preoperatively performed angiography in the operating room, the surgical procedure can be started within minutes and the A. femoralis catheter is left in situ for intraoperative angiography. A CT scan can be performed immediately before and after the angiography and operation. Therefore, rebleeding or hydrocephalus and the need for hematoma evacuation or external ventricular drainage (EVD) can be defined. The intraoperative DSA can exclude any residual aneurysm that would involve a risk of rebleeding.

Immediately after diagnostic DSA in the angiography suite or 24 hours later, all patients underwent a follow-up CT scan.

In-hospital rebleeding after SAH was defined in patients experiencing a progression of the $\mathrm{SAH}$, confirmed in followup CT, after admission to our department. The time from the initial SAH to the rebleeding was defined by the time from initial CT (this could be performed either in the referral or in our hospital) with SAH to the repeated CT that confirmed rebleeding. EVD was placed in any patient who presented with depressed loss of consciousness (LOC) and documented intraventricular hemorrhage or acute hydrocephalus. Transcranial Doppler sonography was performed daily. In cases of suspicion of cerebral vasospasm, CTA including perfusion measurements and DSA were performed. The management of cerebral vasospasm was undertaken using the guidelines of our department. 20,21

Based on preoperative evaluation, angiographic findings, clinical characteristics, and the discussion with our interventional neuroradiologists, the treatment method of the ruptured aneurysm was determined depending on size, shape, location of the aneurysm, and the patient's condition. The occlusion of the aneurysm was performed within 24 hours after admission. If the patient's neurologic status was poor (H\&H grade 5), an EVD for cerebrospinal fluid (CSF) drainage and ICP monitoring was implanted before neurologic assessment after discontinued sedation. Occlusion of the aneurysm in patients with poor neurologic status (grade 5) after SAH was planned after documentation of clinical improvement. Survivors were transferred to rehabilitation. Clinical follow-up examination was performed at our outpatient clinic within 3 months, after 6 months, and then annually until the attending neurosurgeon stopped further consultations due to the patient's stable state. The neurologic status after treatment included the assessment of GCS, focal neurologic deficits, and, if possible, a neuropsychological evaluation. ${ }^{23,24}$

\section{Results}

Our 28 patients who had rebleeding ranged in age from 39 to 75 years (mean: $58 \pm 10$ years) and included 16 women (57\%) and 12 men (43\%) (-Table 1). Of these 28 patients, $20(71 \%)$ died after rebleeding of the ruptured aneurysm after admission. The numbers of rebleeding episodes were one episode in 16 cases (57\%), two episodes in 8 (29\%), and three episodes in 4 patients (14\%). Among the 12 patients (43\%) with two or three rebleeding episodes, outcomes were worse: 10 patients (36\%) died and 2 (7\%) had GOS 3 (mRS: 5). Rebleeding was documented during the first 4 hours after initial SAH in 15 patients (54\%), within the first 24 hours in 6 (21\%), and within 48 hours in 1 (4\%). Additional episodes of rebleeding occurred in two patients (8\%) within 72 hours after admission and in two (7\%) within 4 days, one (4\%) within 5 days, and one (4\%) after 9 days.

The location of the aneurysm included anterior cerebral artery (ACA) and anterior communicating artery (AComA) in 13 patients (46\%), middle cerebral artery (MCA) in 9 (32\%), internal carotid artery in 4 (14\%), and vertebrobasilar circulation in 2 (7\%) (-Table 2). Eight patients (29\%) had more than one aneurysm, and 16 (57\%) had polylobular types.

Analyzing hemodynamic parameters during rebleeding, we obtained MAP and SBP before and during rebleeding. In this series, MAP was $98 \pm 11 \mathrm{~mm} \mathrm{Hg}$ at arrival at the emergency department and $88 \pm 10 \mathrm{~mm} \mathrm{Hg}$ before rebleeding. In three cases (patients 5, 18, and 28), MAP values over the mean value of the entire group could not be documented, due to manipulation maneuvers such as implantation of catheters. During rebleeding, MAP dramatically increased by a mean of $36 \mathrm{~mm} \mathrm{Hg}$ to $124 \pm 22 \mathrm{~mm} \mathrm{Hg}$ and decreased gradually to $85 \pm 11 \mathrm{~mm} \mathrm{Hg}$ after the ictus within the following first hour ( - Table 3 ).

Of these rebleeding patients, 24 (85\%) underwent ICP monitoring immediately after admission, with acute mean ICP increases of up to $83 \pm 20 \mathrm{~mm} \mathrm{Hg}$ during rebleeding. Subsequently, increased blood pressure during rebleeding reversed spontaneously or was lowered to $86 \pm 12 \mathrm{~mm} \mathrm{Hg}$ with antihypertensive medication after the episode. Rebleeding caused epileptic seizures in three patients (10\%). Additionally, rebleeding episodes occurred in six patients (21\%) during or shortly after DSA and in six patients (21\%) during or just after referral to our hospital or during transportation to radiologic evaluation or to the ICU. Three patients (10\%) had been taking aspirin for cardiac disease. In one patient, whose aneurysm was confirmed in the DSA and no signs of SAH in 


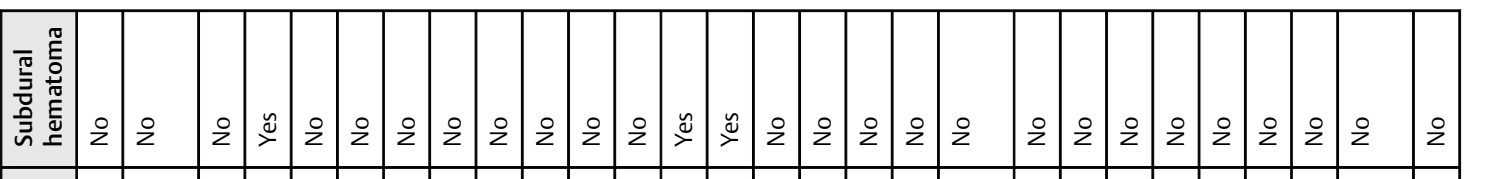

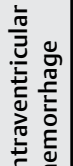

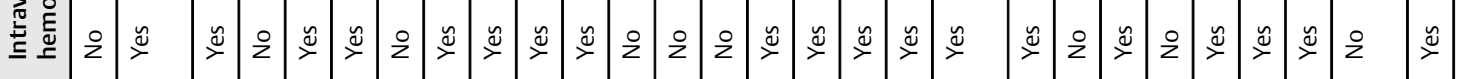

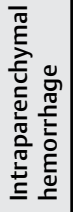

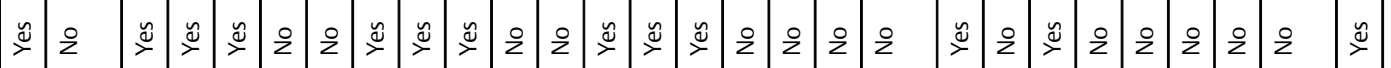

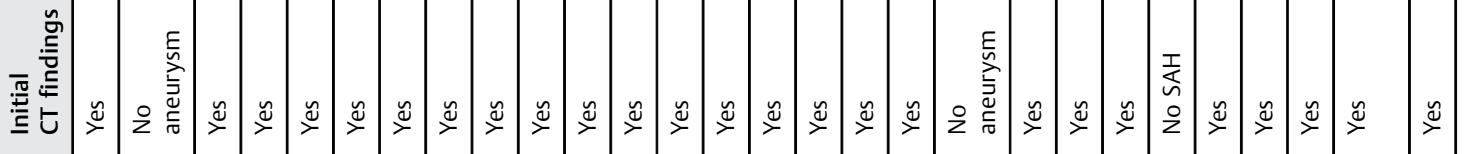

䈉

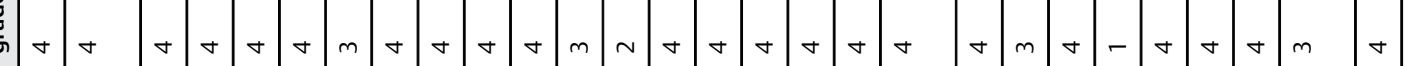

๖

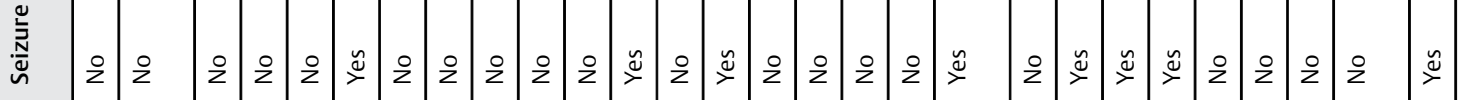

赵

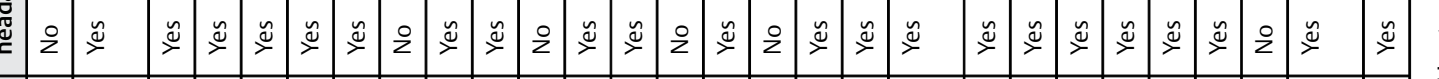

こับ

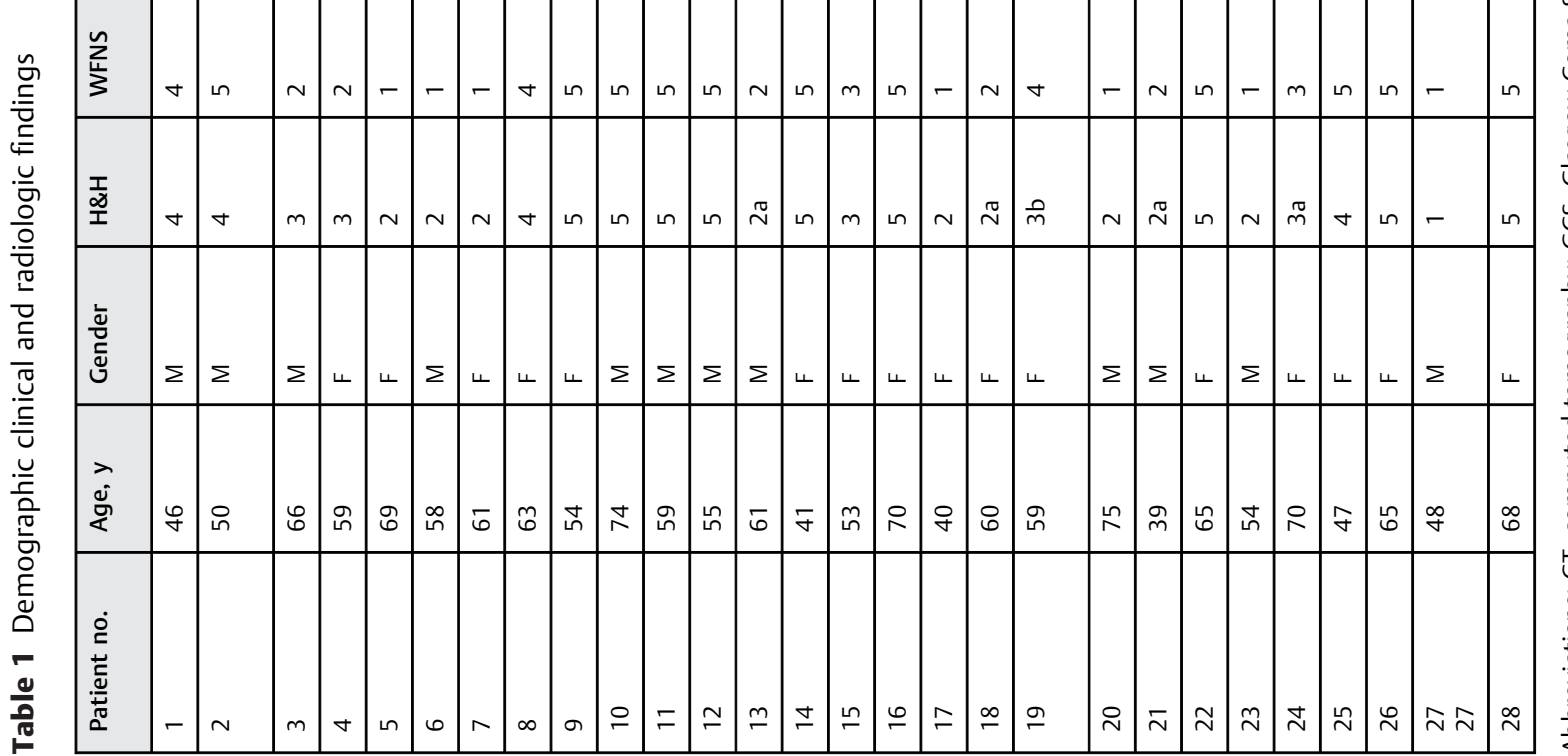

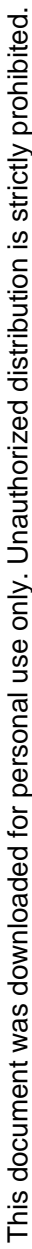




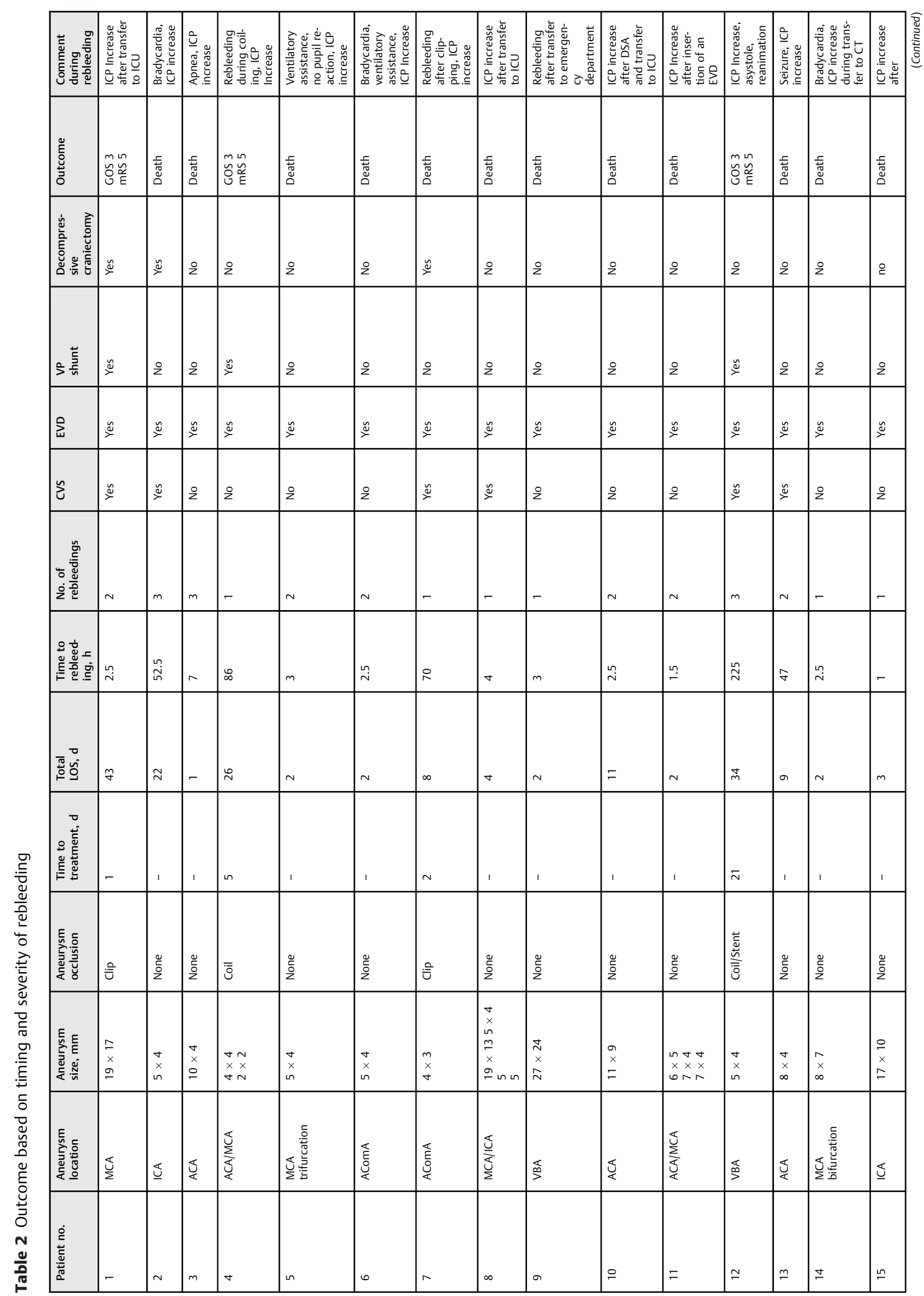




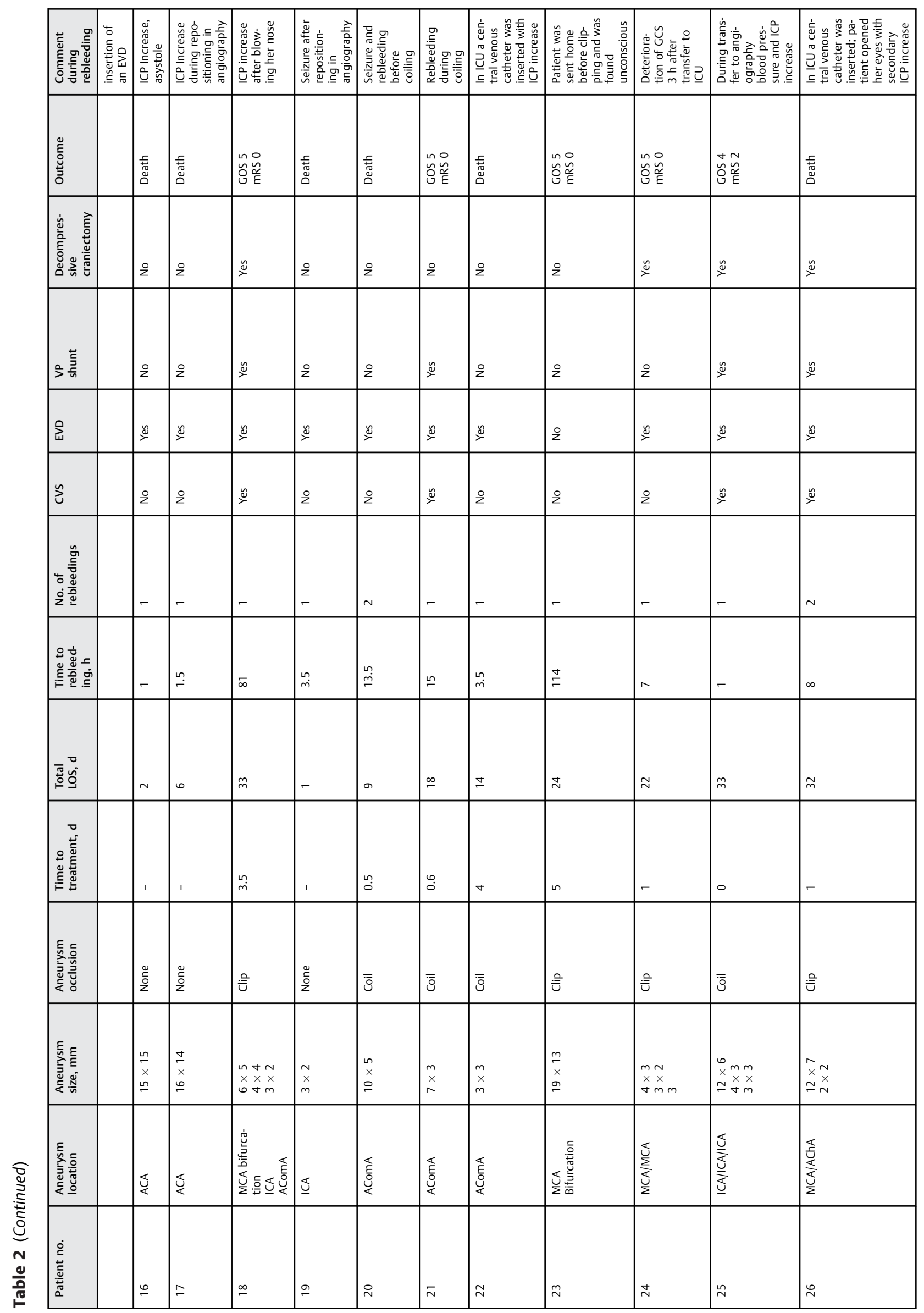




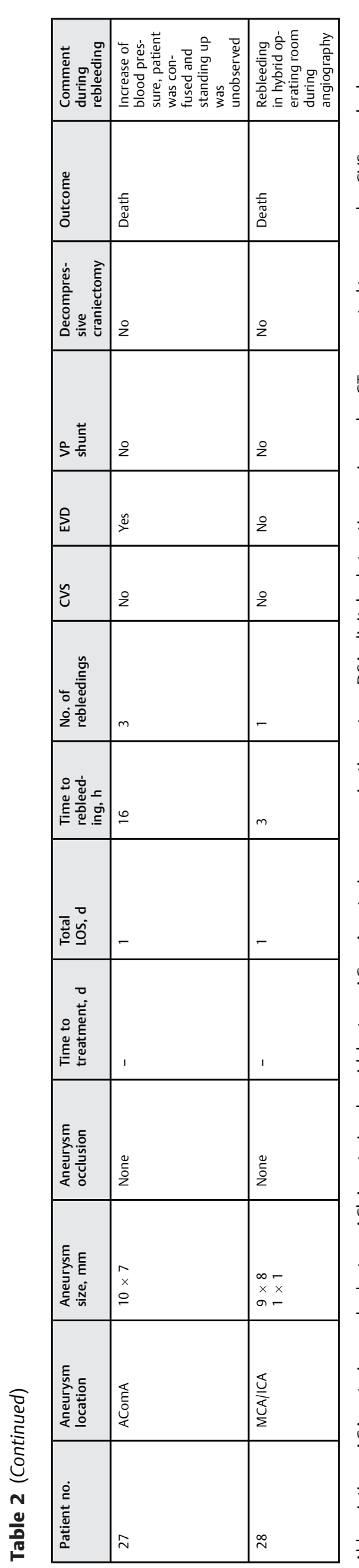

the CT could be observed, rebleeding occurred prior to elective clipping. This patient had sentinel symptoms corresponding to a first bleeding episode days before his initial admission. He had been found unconscious at home, and traces of a ruptured right MCA aneurysm were detected after rebleeding. The patient then underwent emergency clipping and evacuation of an intracerebral hematoma.

Mobilization of the patient and procedures including invasive techniques such as insertion of central venous catheters prior to the occlusion of the ruptured aneurysm were restricted to a minimum; nevertheless, rebleeding was observed in two patients during insertion of central venous catheters and six patients during DSA. Moreover, in one patient, rebleeding occurred during sneezing in the ICU, in two patients while standing up confused in the ICU (one had his eyes open during the procedure), in one patient rebleeding was documented during the emergency CT when his GCS suddenly dropped, and in seven patients it happened during circulatory arrest. Here we must state, however, that rebleeding could have caused the cardiac arrest.

\section{Clinical Findings during Rebleeding}

Further initial clinical findings at arrival at the emergency department in our institution or the primary hospital were sentinel headaches in 22 patients (79\%), LOC in 19 (68\%), and seizure as a primary symptom in 8 (29\%) (-Table 1). As documented on admission, WFNS grade was I in seven patients (25\%), grade II in five (18\%), and grade III in two (7\%). Higher grades, IV and V, were observed in $3(11 \%)$ and 11 patients (39\%), respectively (-Table $\mathbf{1}$ ).

On admission, $\mathrm{H} \& \mathrm{H}$ grades were grade I in one patient (4\%), grade II in nine (32\%), grade III in five (18\%), established earlier with mild focal neurologic deficits, grade IV in four (14\%), and grade $\mathrm{V}$ in nine (32\%). Consciousness in terms of GCS scores was rated $13-15$ in 14 patients (50\%), 9 in $1(4 \%)$, and $\leq 7$ in 5 (18\%). The remaining eight patients (29\%) arrived intubated at the emergency department (GCS 3) (-Table 1).

On initial CT findings, Fisher grades were assigned according to the extent of SAH (-Table 1). The majority, 23 (82\%) of the patients, presented with a Fisher grade IV on the initial CT scan, whereas 4 (14\%) presented with a Fisher grade III, and 1 (4\%) presented with grade II. On the initial CT, 2 patients (7\%) did not show an aneurysm as the cause of the SAH. The hemorrhage on the admission CT was determined to be intraparenchymatous in 14 patients (50\%) and intraventricular in 19 (68\%). These findings correlate with the high number of cases classified as Fisher grade IV. Additional subdural hematoma was found in three cases (11\%). On admission day, 17 patients (61\%) underwent DSA, their neurologic status permitting this procedure.

\section{Treatment}

Treatment was achieved in a total of 12 patients (43\%) by either coiling (6 patients) or clipping (6 patients). This treatment of the ruptured aneurysm was delivered within 48 hours after ictus in 6 patients (i.e., 3 patients within 24 hours and 3 between 24 and 28 hours). Five patients (18\%) 
214 In-Hospital Rebleeding of Rupture of Intracranial Aneurysms Kienzler et al.

Table 3 Blood pressure values at admission and prior, during, and after rebleeding

\begin{tabular}{|c|c|c|c|c|c|c|c|c|}
\hline \multirow[b]{2}{*}{ Patient no. } & \multicolumn{2}{|c|}{ At admission } & \multicolumn{2}{|c|}{ Before rebleeding } & \multicolumn{2}{|c|}{ During rebleeding } & \multicolumn{2}{|c|}{ After rebleeding } \\
\hline & SBP/DBP & MAP & SBP/DBP & MAP & SBP/DBP & MAP & $\mathrm{SBP} / \mathrm{DBP}$ & MAP \\
\hline 1 & $160 / 70$ & 97 & $140 / 60$ & 84 & $210 / 80$ & 119 & $100 / 50$ & 65 \\
\hline 2 & $140 / 100$ & 112 & $140 / 60$ & 84 & $210 / 80$ & 119 & $150 / 60$ & 87 \\
\hline 3 & $173 / 89$ & 114 & $120 / 60$ & 78 & $210 / 100$ & 133 & $140 / 60$ & 84 \\
\hline 4 & $123 / 71$ & 86 & $130 / 60$ & 81 & $220 / 170$ & 185 & $140 / 60$ & 84 \\
\hline 5 & $170 / 100$ & 121 & $170 / 80$ & 107 & $220 / 120$ & 150 & $140 / 80$ & 98 \\
\hline 6 & $140 / 80$ & 98 & $140 / 70$ & 91 & $160 / 70$ & 97 & $140 / 70$ & 91 \\
\hline 7 & $140 / 70$ & 91 & $120 / 70$ & 85 & $200 / 80$ & 116 & $140 / 60$ & 84 \\
\hline 8 & $150 / 80$ & 101 & $130 / 60$ & 81 & $140 / 70$ & 91 & $110 / 60$ & 75 \\
\hline 9 & $170 / 80$ & 107 & $120 / 60$ & 78 & $210 / 120$ & 147 & $150 / 60$ & 87 \\
\hline 10 & $150 / 90$ & 108 & $150 / 70$ & 94 & $180 / 80$ & 110 & $140 / 70$ & 91 \\
\hline 11 & $132 / 83$ & 98 & $140 / 80$ & 98 & $160 / 90$ & 111 & $120 / 60$ & 78 \\
\hline 12 & $120 / 60$ & 78 & $130 / 70$ & 88 & $170 / 100$ & 121 & $120 / 80$ & 92 \\
\hline 13 & $140 / 80$ & 98 & $100 / 60$ & 72 & $160 / 90$ & 111 & $120 / 60$ & 78 \\
\hline 14 & $100 / 70$ & 79 & $120 / 80$ & 92 & $150 / 100$ & 115 & $120 / 80$ & 92 \\
\hline 15 & $130 / 80$ & 95 & $140 / 70$ & 91 & $210 / 140$ & 161 & $140 / 70$ & 91 \\
\hline 16 & $160 / 80$ & 104 & $120 / 50$ & 71 & $200 / 80$ & 116 & $180 / 70$ & 103 \\
\hline 17 & $140 / 60$ & 84 & $120 / 50$ & 71 & $190 / 80$ & 113 & $140 / 50$ & 77 \\
\hline 18 & $190 / 60$ & 99 & $140 / 110$ & 119 & $190 / 120$ & 141 & $130 / 80$ & 95 \\
\hline 19 & $130 / 80$ & 95 & $120 / 80$ & 92 & $240 / 140$ & 170 & $140 / 100$ & 112 \\
\hline 20 & $140 / 80$ & 98 & $140 / 60$ & 84 & $200 / 80$ & 116 & $80 / 40$ & 52 \\
\hline 21 & $100 / 60$ & 72 & $130 / 70$ & 88 & $210 / 120$ & 147 & $120 / 60$ & 78 \\
\hline 22 & $155 / 66$ & 93 & $140 / 60$ & 84 & $150 / 70$ & 94 & $130 / 50$ & 74 \\
\hline 23 & $140 / 100$ & 112 & $130 / 70$ & 88 & $170 / 90$ & 114 & $120 / 60$ & 78 \\
\hline 24 & $160 / 90$ & 111 & $139 / 76$ & 95 & $190 / 100$ & 127 & $150 / 60$ & 87 \\
\hline 25 & $130 / 80$ & 95 & $140 / 70$ & 91 & $170 / 80$ & 107 & $130 / 70$ & 88 \\
\hline 26 & $120 / 80$ & 92 & $120 / 60$ & 78 & $160 / 90$ & 111 & $130 / 70$ & 88 \\
\hline 27 & $140 / 80$ & 98 & $120 / 80$ & 92 & $130 / 90$ & 102 & $120 / 80$ & 92 \\
\hline 28 & $160 / 80$ & 104 & $150 / 80$ & 101 & $200 / 90$ & 123 & $150 / 80$ & 101 \\
\hline $\begin{array}{l}\text { Mean SBP or } \\
M A P \pm S D, m m ~ H g\end{array}$ & $142 \pm 20$ & $98 \pm 11$ & $132 \pm 13$ & $88 \pm 10$ & $186 \pm 27$ & $124 \pm 22$ & $131 \pm 18$ & $85 \pm 11$ \\
\hline
\end{tabular}

Abbreviations: DBP, diastolic blood pressure, $\mathrm{mm} \mathrm{Hg}$; MAP, mean arterial blood pressure, $\mathrm{mm} \mathrm{Hg}$; SBP, systolic blood pressure, $\mathrm{mm} \mathrm{Hg}$; SD, standard deviation.

received treatment within 5 days due to the severity of their SAH or other clinical findings. Finally, one case (4\%) underwent endovascular occlusion after 21 days after conservative therapy and rehabilitation due to the initially poor clinical situation of the comatose patient. Acute hydrocephalus after SAH was documented in 24 cases (86\%).

Of 26 patients (93\%) who had EVD, 21 (75\%) showed persisting hydrocephalus signs and then underwent ventriculoperitoneal shunting. Decompressive craniectomy was performed in seven patients (25\%) presenting with severe edema and increased ICP signs. Six of the seven patients who underwent decompressive craniectomy survived. In three patients $(11 \%)$ a bacterial infection could be documented. One of them presented with a subcutaneous and skull flap infection. In the other two patients, pneumonia was documented in one case and cystitis in the other. Cerebral vasospasm was documented in 10 cases (36\%). When cerebral vasospasm occurred, standard therapy with hypertension and chemical angioplasty with nimodipine was performed according to our institution's protocol. ${ }^{20,23}$ Despite maximal therapy, nine patients developed permanent ischemia and permanent neurologic deficits. Of the 10 patients with severe vasospasm, 5 survivors developed delayed cerebral ischemic deficits and 5 patients died. 


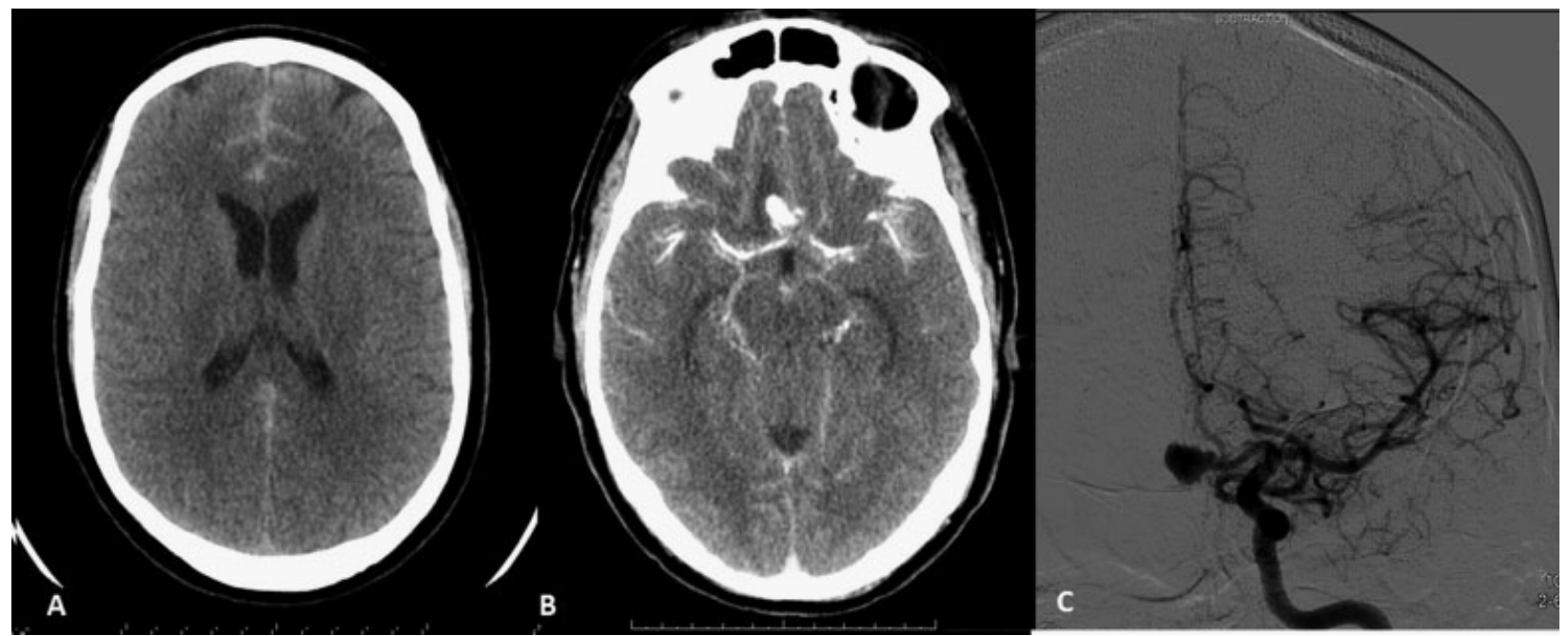

Fig. 1 Case 1: Initial aneurysmal subarachnoid hemorrhage. A 48-year-old man presenting with headaches, vertigo, nausea, and loss of consciousness caused by anterior communicating artery (AComA) aneurysm. Computed tomography (CT) scans showing rupture of AComA aneurysm in (A) axial view and (B) CT angiography view. (C) Digital subtraction angiography confirmed the $7 \times 10 \mathrm{~mm}$ aneurysm.

The ICU stay after aneurysm occlusion averaged $18 \pm 10$ days. The overall hospital stay (including ICU and ward stay) averaged $25 \pm 14$ days. Finally, all eight patients (29\%) who survived were transferred to neurologic rehabilitation after treatment.

\section{Outcomes of Rebleeding after Aneurysmal SAH}

The mortality after rebleeding in our series was $71 \%$. In comparison, overall mortality was $7.8 \%$ for the total of the $243 \mathrm{SAH}$ patients. At the last follow-up examination (mean: 19.7 months; range: 8-39 months), 4 (14\%) of the 28 rebleeding patients had a GCS score of 15 without any neurologic deficits; 2 patients (7\%) had a GCS score of 14 and showed a persisting hemiparesis, and 2 other patients (7\%) had GCS scores between 10 and 12. GOS 5 (mRS: 0 ) was found in four patients, one had GOS 4 (mRS: 2), and three patients had GOS 3 (mRS: 5) and presented with severe neurologic deficits (e.g., hemiplegia, aphasia) (- Table 2). Only one patient had resumed the previous job and activities.

\section{Illustrative Cases}

\section{Case 1}

A 48-year-old man was referred to our hospital after presenting with headaches, vertigo, nausea, and loss of consciousness (LOC). After admission a CT scan demonstrated SAH after rupture of an anterior communicating artery (AComA) aneurysm. DSA confirmed these findings ( - Fig. 1A, B). On neurologic examination, the patient presented with a GCS score of 15 , meningism, and had no neurologic deficits. The emergency DSA demonstrated a $10 \times 7 \mathrm{~mm}$ AComA aneurysm(-Fig. 1C). After his transfer to the ICU, the patient became increasingly confused and complained of headache. On day 2 after bleeding, he stood up, with blood pressure

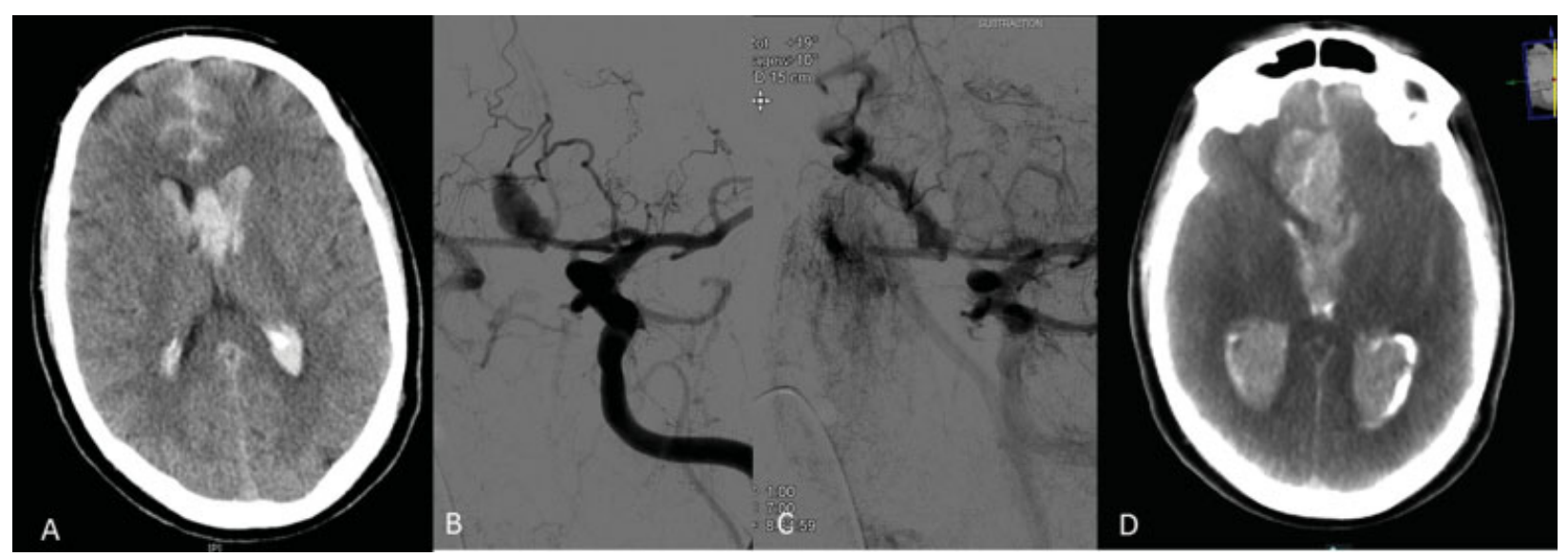

Fig. 2 Case 1: Rebleeding. (A) Computed tomography (CT) scan showing rebleeding immediately after increased blood pressure values. (B, C) Active rerupture with rebleeding of the aneurysm documented during digital subtraction angiography (DSA). (D) CT scan after DSA showed severe intraventricular and intraparenchymatous rebleeding with progressive occlusive hydrocephalus. 


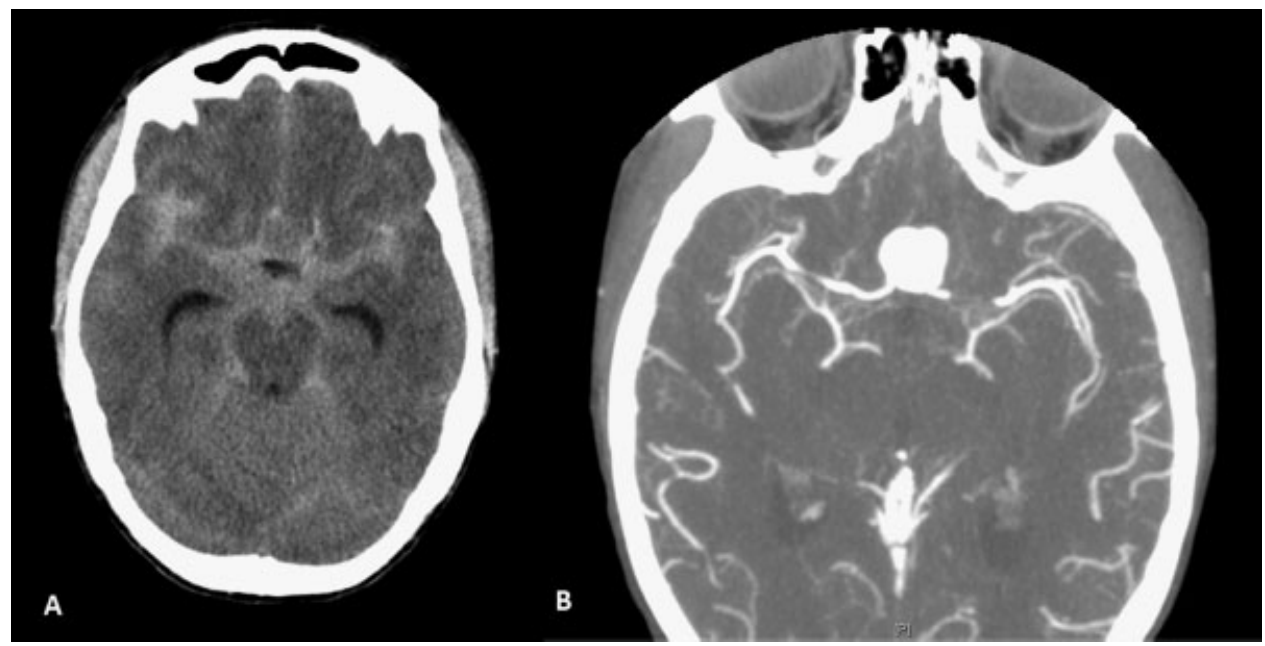

Fig. 3 Case 2: Initial aneurysmal subarachnoid hemorrhage (SAH). (A) Initial computed tomography scan shows the SAH in the anterior basal cisterns. (B) Digital subtraction angiography demonstrating anterior communicating aneurysm.

peaking at $170 / 99 \mathrm{~mm} \mathrm{Hg}$. With an emergency CT head scan showing a rebleeding with intraventricular blood clot and the beginning of an occlusive hydrocephalus, he was transferred back to the ICU with a documented deteriorating GCS score. After being intubated and mechanically ventilated and considering the CT findings, the patient had an EVD implanted that showed an ICP which was elevated to $100 \mathrm{~mm} \mathrm{Hg}$.

The follow-up CT scan the day after admission showed a severe second rebleeding with progressive hydrocephalus (-Fig. 2A). The patient was transferred to an emergency angiography and endovascular treatment. During the coiling of the aneurysm, a rerupture with active bleeding was documented (-Fig. 2B, 2C). The CT scan after DSA showed an increase of intraventricular and intraparenchymal blood collection with progressive occlusive hydrocephalus (-Fig. 2D). The ICP remained unchanged ( $~ 80 \mathrm{~mm} \mathrm{Hg}$ ) during the procedure and the endovascular treatment was interrupted. The patient was transferred back to the ICU and died.

\section{Case 2}

Noting 2 days of decreasing visual acuity and left scotoma, this 40-year-old woman received a diagnosis of optic nerve pathology by the ophthalmologist and was referred to the eye clinic at our institution. When the patient suddenly experienced a severe headache, with nuchal pain and stiffness, an emergency CT scan was performed and showed an acute SAH (-Fig. 3A, B). In our emergency department, the initial neurologic examination showed a GCS score of 15 . The patient was anguished, but no additional focal neurologic deficits could be observed. With a second clinical evaluation demonstrating GCS 11, and considering the rapid neurologic deterioration and CT findings, the patient underwent emergency intubation and EVD placement. The emergency DSA detected a $16 \times 14 \mathrm{~mm}$ AComA aneurysm(-Fig. 4A, B). During transfer onto the angiography table, her ICP elevated to $100 \mathrm{~mm} \mathrm{Hg}$. There, in the angiography suite, the repeated CT confirmed an intraventricular and intraparenchymatous

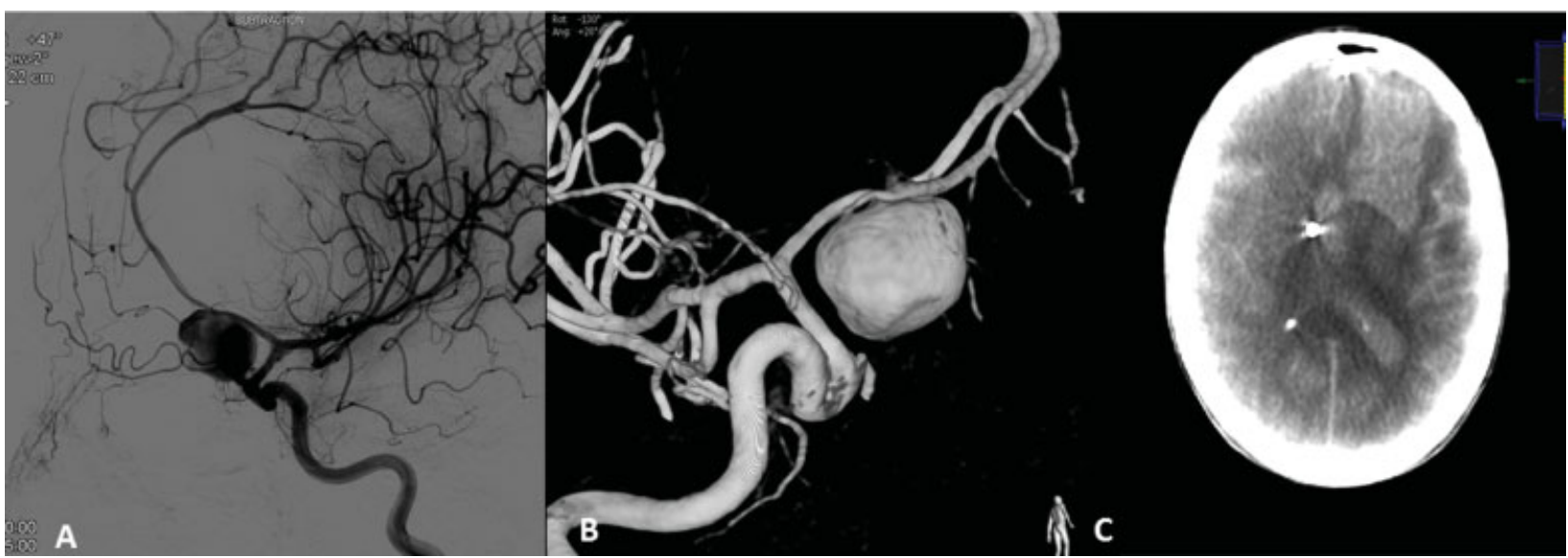

Fig. 4 Case 2: Rebleeding. (A) Digital subtraction angiography (DSA) confirmed an aneurysm (14 $\times 16 \mathrm{~mm})$ located at the anterior communicating artery. (B) Three-dimensional reconstruction showing aneurysm configuration and relationship of two A2 segments. (C) Computed tomography scan after DSA demonstrating massive intraparenchymatous and intraventricular rebleeding with progressive occlusive hydrocephalus. 


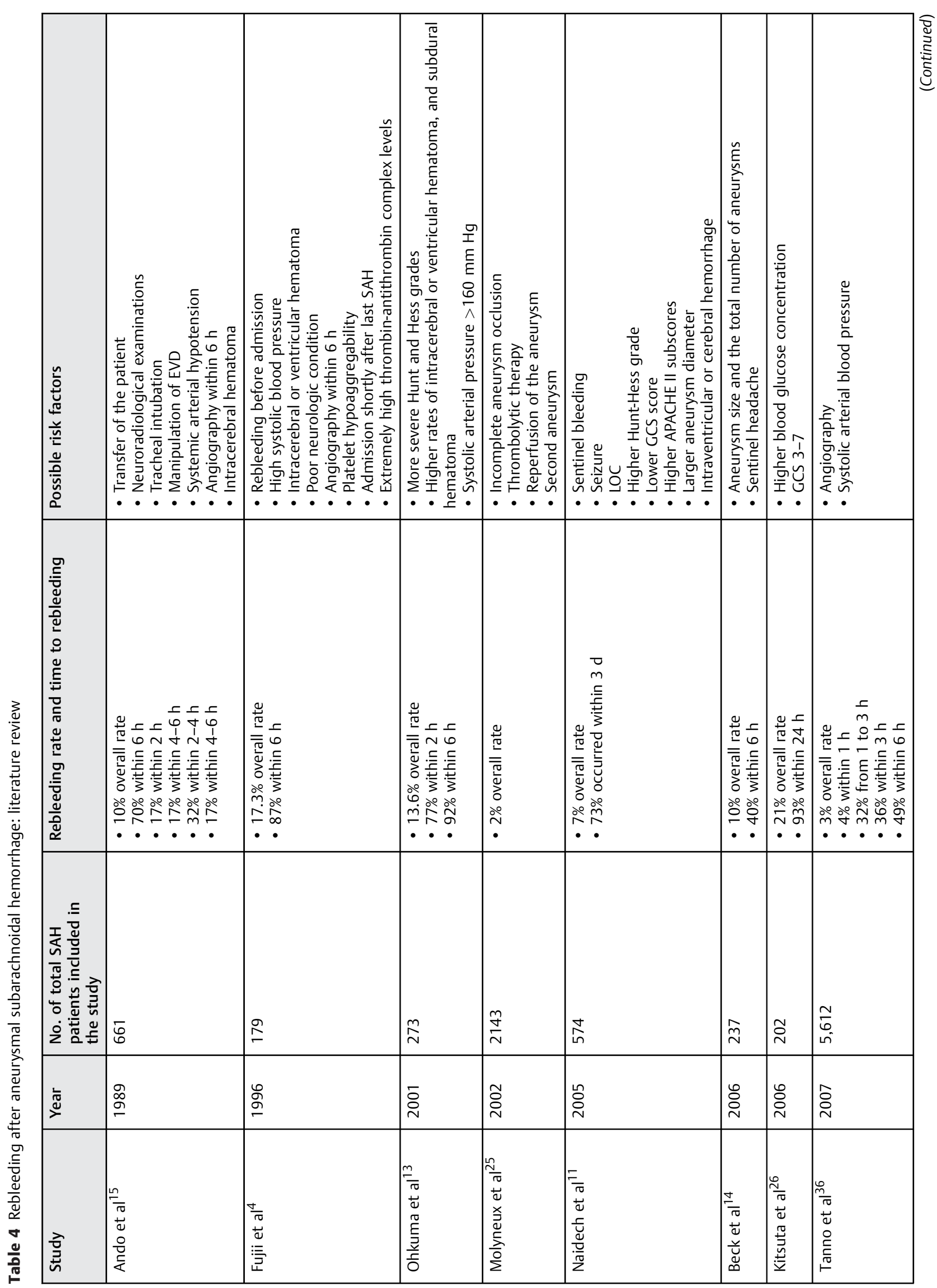




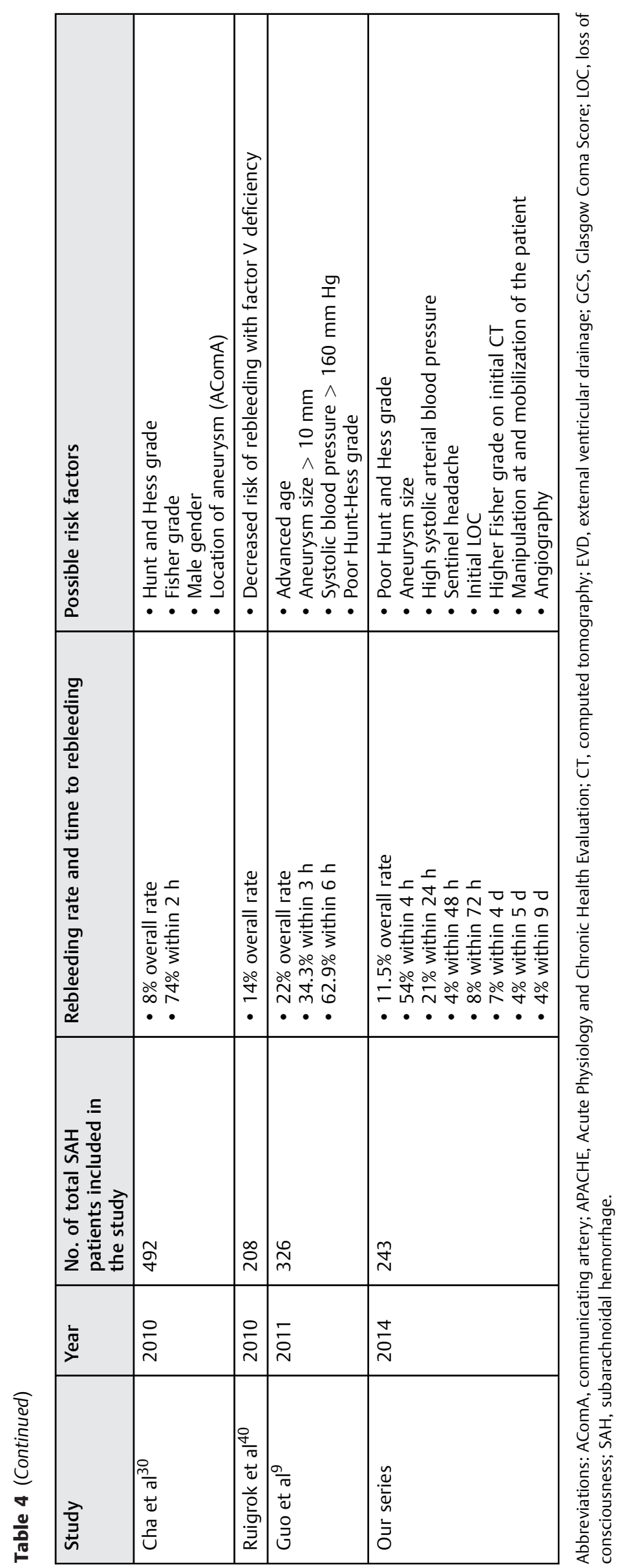


rebleeding associated with progressive occlusive hydrocephalus (-Fig. 4C). When the ICP increased and became intractable, the procedure was aborted and the patient was returned to the ICU. With no clinical improvement after aggressive medical treatment, the therapy was discontinued and the patient died after a prior diagnosis of brain death.

\section{Discussion}

Our study demonstrated the high incidence of in-hospital rebleedings: 28 of 243 patients after acute aneurysmal SAH. We assume that our high rebleeding rate at least partially is due to the improvements of the awareness of our population, of the rescue procedures, and the time until diagnosis after admission in Switzerland: Simply a higher number of rebleeding events reach the hospital compared with the past. Risk factors for rebleeding in our series included high systolic blood pressure, sentinel headache, initial LOC, poor H\&H grade, higher Fisher grade on initial CT, aneurysm size, angiography, and manipulation and mobilization of the patient.

In other studies the rebleeding rate ranged from 2 to $22 \%$ and was in line with the $11.5 \%$ in our series. The rate was as low as $2 \%$ in the International Subarachnoid Aneurysm Trial (ISAT) trial but remarkably higher with $17.3 \%$ in the study of Fujii et al, ${ }^{4}$ with $13.6 \%$ in the study of Ohkuma et al, ${ }^{13}$ with $21 \%$ in the study of Kitsuta et al, ${ }^{26}$ and with $22 \%$ in the study of Guo et al. ${ }^{9}$

Rebleeding typically occurred within a few hours after the initial SAH ( - Table 4): $:^{27,28}$ In our series, it occurred within 4 hours in 15 patients (54\%), within the first 24 hours in $6(21 \%)$, within 48 hours in 1 (4\%), and within 72 hours in 2 (8\%). The remaining four cases had rebleeding as follows: two (7\%) within 4 days, one (4\%) within 5 days, and one (4\%) case after 9 days. These patients also rebleed later. Because of the severity of their neurologic presentation (WFNS grade IV or V), no early aneurysms occlusion was intended, but observation was used to identify any potential neurologic improvement. While earlier studies had shown that rebleeding occurs after 1 week, ${ }^{29}$ more recent series have reported much earlier rebleeding: within 6 hours from SAH onset in $87 \%$ of rebleedings in the study of Fujii et al, ${ }^{4} 63 \%$ in the study of Guo et al, ${ }^{9}$ and within the first 2 hours in $74 \%$ in the study of Cha et $\mathrm{al}^{30}$ (-Table 4). Therefore, rapid diagnosis and transfer of SAH patients to the hospital are as essential to improve the outcome as the initial treatment to mitigate the rebleeding risk and as cardiopulmonary stabilization. In our series, only six patients (21\%) underwent treatment of the ruptured aneurysm within 48 hours after ictus: three (11\%) within the first 24 hours and three (11\%) between 24 and 28 hours after $\mathrm{SAH}$.

\section{Risk Factors}

CTA is not only considered less likely to trigger reruptures than conventional DSA but can also be performed immediately after confirmation of SAH. ${ }^{31}$ In our series, all patients underwent CT and CTA. However, there are an increasing number of reports on rebleeding during CTA as documented by extravasation of contrast medium during CTA. ${ }^{32}$
The studies reported by Ando, ${ }^{15}$ Fujii, ${ }^{4}$ Tanno, ${ }^{36}$ Kusumi, ${ }^{12}$ and Komiyama and their coauthors, ${ }^{34}$ as well as our own, confirm that angiography, particularly within 3 to 6 hours after $\mathrm{SAH}$, is a risk factor for rebleeding. In our series, $61 \%$ of the patients underwent DSA within 6 hours. Aoyagi and Hayakawa suggest that rebleeding during DSA could be caused by the short but rapid increase in transmural pressure by injection of contrast agent. ${ }^{35}$ Others report that high systolic blood pressure is another risk factor for rebleeding. ${ }^{4,9,10,30,36}$ The increased MAP after rerupture of the aneurysm might be interpreted as a compensatory effect to ensure sufficient CPP or as a consequence of the Cushing reflex.

The upper limit, according to our series and that of others, seems to be an arterial systolic pressure of $140 \mathrm{~mm} \mathrm{Hg}$. Given that an increase in arterial pressure or a decrease in CSF pressure results in increased transmural pressure, this creates a pressure gradient equivalent to the CPP that varies in proportion with the MAP. Hypothetically, CSF drainage through the insertion of an EVD could cause an increase in the transmural pressure through a decrease in ICP and aggravate the risk of rebleeding. However, there is no current evidence to support this. ${ }^{37,38}$

Studies investigating antifibrinolytic therapy to reduce rebleeding have failed to clearly demonstrate overall therapeutic benefit. Although short-course antifibrinolytic therapy may play a certain role before initial aneurysm repair, current data are insufficient. ${ }^{1,8}$

Larsen et al hypothesized that patients with SAH develop a coagulopathy characterized by reduced clot stability soon after the initial bleeding. ${ }^{24}$ For patients with aneurysmal $\mathrm{SAH}$, a dynamic clot lysis assay was performed. Markers of fibrinolysis and clot stabilizers were taken from blood samples within and $>6$ hours after bleeding onset. The results were compared with blood samples from age- and sex-matched healthy controls. Patients demonstrated significantly reduced clot stability during the first 6 hours after initial bleeding. Specifically, within the first 6 hours, fibrinolytic activity and the inhibitors of fibrinolysis increased, whereas the modulators of fibrinolysis were reduced or inactivated. This is a probable consequence of the activated fibrinolysis in combination with reduced or inactivated factor XIII and thrombin-activatable fibrinolysis inhibitor. ${ }^{24}$

Another risk factor found in our series and in those of others included an initial poor neurologic presentation with a high $\mathrm{H} \& \mathrm{H}$ grade. ${ }^{4,9-11,13,26,30}$

On admission to the emergency department, WFNS grades IV and $\mathrm{V}$ were seen in $50 \%$ of patients, $\mathrm{H} \& \mathrm{H}$ grades III to $\mathrm{V}$ were documented in $64 \%$, and GCS scores $\leq 9$ affected $51 \%$ of these cases.

Interestingly, intracerebral and intraventricular hematomas have occurred as risk factors in multiple stud-

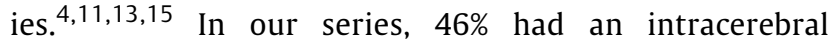
hematoma; $68 \%$ presented an intraventricular hemorrhage. This might have been a manifestation of a major perforation, and therefore a bigger leak could earlier lead to rebleeding. However, intracerebral or intraventricular 
hematoma correlates with Fisher grade. Given that $82 \%$ of our patients presented with a Fisher grade IV on the initial CT scan, we concluded that the higher Fisher grade was a risk factor.

The documented LOC in $68 \%$ of our patients coincided with a similar finding by Naidech et $\mathrm{al}^{11}{ }^{11}$ who indicated LOC as a risk factor. Like Beck et al, ${ }^{14}$ who noted sentinel headache as a risk factor for rebleeding, $79 \%$ of our patients also had this type of headache.

In a literature review, Larsen et al explain that rebleeding is a complex and multifactorial event involving hemostasis and pathophysiologic and anatomical factors. ${ }^{37}$ Although increased risk of rebleeding is associated with poor clinical condition (e.g., high H\&H grade) on arrival, conflicting data exist regarding the characteristics of the aneurysm (e.g., size, location).

In contrast, a large aneurysm diameter was deemed a risk factor in several studies by Naidech et al, ${ }^{11}$ Beck et al, ${ }^{14}$ Guo et $\mathrm{al}^{9}{ }^{9}$ and our series. Specifically, $65 \%$ of our $\mathrm{SAH}$ patients had a rebleeding aneurysm with a diameter $>6 \mathrm{~mm}$. In other series, aneurysm number ${ }^{14}$ and AComA location ${ }^{30}$ were relevant for this risk. We confirmed these observations, finding that $29 \%$ of our patients had more than one aneurysm and detecting $46 \%$ of aneurysms at ACA and AComA and 32\% at the MCA. In a subgroup analysis, Cong et $\mathrm{al}^{10}$ showed that posterior circulation aneurysms have a significantly higher rebleeding rate (34\%) than the nonposterior circulation aneurysms (11.4\%). Larsen and Astrup showed that no difference in the rebleeding rate between anterior and posterior circulation aneurysms can be detected. ${ }^{37}$

Regarding sex, our series included slightly more females like other reports, ${ }^{39}$ whereas Cha et $\mathrm{al}^{30}$ and Cong et al ${ }^{10}$ claimed that male sex is a risk factor for rebleeding.

The transfer and manipulation of the patient, such as the placement of a central venous catheter or the tracheal intubation and implantation of an $\mathrm{EVD}^{15}$ were often followed by SAH rebleeding. In our 26 patients (93\%) who underwent the placement of EVD, we explain this finding with a high Fisher grade (IV) bleeding in which ventricular drainage was used to prevent hydrocephalus. Therefore rebleeding was associated with the indication, not the insertion, of the EVD itself.

Other authors report on mortality and morbidity of rebleeding after aneurysmal SAH as well. Similar results in terms of outcome (GOS) were reported by Guo et $\mathrm{al}^{9}$ with good recovery in $21 \%$ of patients, moderate disability in $13 \%$, severe disability in $11 \%$, and $7 \%$ who remained in a persistent vegetative state. The overall mortality was $47 \%$ in their series. At 3-month follow-up after rebleeding, Tanno et al $^{36}$ reported $18 \%$ of the patients with good recovery or condition, $8 \%$ with moderate disability, and $7 \%$ with severe disability. The mortality was $60 \%$. Another 3-month follow-up study by Naidech et $\mathrm{al}^{11}$ found that rebleeding is associated with a significantly reduced chance of survival with functional independence ( $\mathrm{mRS}$ score $\leq 4$ ). These results are similar to earlier studies, such as Ando et $\mathrm{al}^{15}$ in 1989, who reported a $65 \%$ mortality rate.

\section{Study Limitations}

One study limitation is the impossibility of a rebleeding assessment before arrival at the hospital. In Switzerland patients normally reach the vascular center hospital within 1 to 2 hours after the first perception of symptoms, a short period during which rebleeding events cannot be recorded. Studies that take account of this phase primarily originate in countries like China where transport may last days until the arrival at a hospital with a vascular neurosurgical department. Another limitation might be that risk factors like a sentinel headache or the initial LOC can be missed by nonstandardized documentation. Moreover, although the incidence is comparatively high, our series is rather small due to the geographic dimensions of Switzerland and the catchment area of our clinic. Finally, our study is literally retrospective due to the fact that rebleeding occurs within the first hours after stroke onset, partially before arrival at the emergency department, and may be diagnosed or confirmed only retrospectively. Our focus was on the description of the rebleeders' symptoms as well as their clinical and radiologic characteristics. Because all patients showing these signs must have special care, we decided not to assess a retrospective control group (i.e., the patients without rebleeding).

\section{Conclusions}

In-hospital rebleeding after SAH is a devastating condition with high mortality and poor outcome in survivors. Given that most rebleeding cases are likely to occur within 7 hours of admission, early occlusion of the aneurysm should be further considered. Based on our findings, we suggest that mobilization of the patient and maneuvers including invasive procedures should be restricted to a minimum during ICU treatment prior to the occlusion of the ruptured aneurysm. According to our experience and the review of the literature, stabilization of blood pressure, avoiding blood pressure peaks, and adequate sedation and analgesia given prior to aneurysm occlusion can be considered preventive strategies against in-hospital rebleeding.

\section{References}

1 Starke RM, Connolly ES Jr; Participants in the International MultiDisciplinary Consensus Conference on the Critical Care Management of Subarachnoid Hemorrhage. Rebleeding after aneurysmal subarachnoid hemorrhage. Neurocrit Care 2011;15(2):241-246

2 Ameen AA, Illingworth R. Anti-fibrinolytic treatment in the preoperative management of subarachnoid haemorrhage caused by ruptured intracranial aneurysm. J Neurol Neurosurg Psychiatry 1981;44(3):220-226

3 Jane JA, Winn HR, Richardson AE. The natural history of intracranial aneurysms: rebleeding rates during the acute and long term period and implication for surgical management. Clin Neurosurg 1977;24:176-184

4 Fujii Y, Takeuchi S, Sasaki O, Minakawa T, Koike T, Tanaka R. Ultraearly rebleeding in spontaneous subarachnoid hemorrhage. J Neurosurg 1996;84(1):35-42

5 Hillman J, Fridriksson S, Nilsson O, Yu Z, Saveland H, Jakobsson KE. Immediate administration of tranexamic acid and reduced incidence of early rebleeding after aneurysmal subarachnoid 
hemorrhage: a prospective randomized study. J Neurosurg 2002; 97(4):771-778

6 Laidlaw JD, Siu KH. Ultra-early surgery for aneurysmal subarachnoid hemorrhage: outcomes for a consecutive series of 391 patients not selected by grade or age. J Neurosurg 2002;97(2): 250-258; discussion 247-249

7 Larsen CC, Eskesen V, Hauerberg J, Olesen C, Romner B, Astrup J. Considerable delay in diagnosis and acute management of subarachnoid haemorrhage. Dan Med Bull 2010;57(4):A4139

8 Starke RM, Kim GH, Fernandez A, et al. Impact of a protocol for acute antifibrinolytic therapy on aneurysm rebleeding after subarachnoid hemorrhage. Stroke 2008;39(9):2617-2621

9 Guo LM, Zhou HY, Xu JW, Wang Y, Qiu YM, Jiang JY. Risk factors related to aneurysmal rebleeding. World Neurosurg 2011;76(3-4):292-298; discussion 253-254

10 Cong W, Zhongxin Z, Tiangui L, Zhang Y, Min H, Chao Y. Risk factors for rebleeding of aneurysmal subarachnoid hemorrhage based on the analysis of on-admission information. Turk Neurosurg 2012; 22(6):675-681

11 Naidech AM, Janjua N, Kreiter KT, et al. Predictors and impact of aneurysm rebleeding after subarachnoid hemorrhage. Arch Neurol 2005;62(3):410-416

12 Kusumi M, Yamada M, Kitahara T, et al. Rerupture of cerebral aneurysms during angiography-a retrospective study of 13 patients with subarachnoid hemorrhage. Acta Neurochir (Wien) 2005;147(8):831-837

13 Ohkuma H, Tsurutani H, Suzuki S. Incidence and significance of early aneurysmal rebleeding before neurosurgical or neurological management. Stroke 2001;32(5):1176-1180

14 Beck J, Raabe A, Szelenyi A, et al. Sentinel headache and the risk of rebleeding after aneurysmal subarachnoid hemorrhage. Stroke 2006;37(11):2733-2737

15 Ando T, Sakai N, Yamada H, et al. Analysis of reruptured cerebral aneurysms and the prophylactic effects of barbiturate therapy on the early stage. Neurol Res 1989;11(4):245-248

16 Fisher CM, Kistler JP, Davis JM. Relation of cerebral vasospasm to subarachnoid hemorrhage visualized by computerized tomographic scanning. Neurosurgery 1980;6(1):1-9

17 Drake C. Report of World Federation of Neurological Surgeons Committee on a Universal Subarachnoid Hemorrhage Grading Scale. J Neurosurg 1988;68(6):985-986

18 Hunt WE, Hess RM. Surgical risk as related to time of intervention in the repair of intracranial aneurysms. J Neurosurg 1968;28(1): $14-20$

19 Colford JM Jr, Wade TJ, Sandhu SK, et al. A randomized, controlled trial of in-home drinking water intervention to reduce gastrointestinal illness. Am J Epidemiol 2005;161(5):472-482

20 Yonekawa Y, Imhof HG, Ogata N, et al. Aneurysm surgery in the acute stage: results of structured treatment. Neurol Med Chir (Tokyo) 1998;38(Suppl):45-49

21 Fandino J, Schuknecht B, Yüksel C, Wieser HG, Valavanis A, Yonekawa Y. Clinical, angiographic, and sonographic findings after structured treatment of cerebral vasospasm and their relation to final outcomes. Acta Neurochir (Wien) 1999;141(7):677-690

22 Fandino J, Taussky P, Marbacher S, et al. The concept of a hybrid operating room: applications in cerebrovascular surgery. Acta Neurochir Suppl (Wien) 2013;115:113-117

23 Stienen MN, Weisshaupt R, Fandino J, et al; Swiss SOS study group. Current practice in neuropsychological outcome reporting after aneurysmal subarachnoid haemorrhage. Acta Neurochir (Wien) 2013;155(11):2045-2051

24 Larsen CC, Sørensen B, Nielsen JD, Astrup J. Reduced clot-stability during the first 6 hours after aneurysmal subarachnoid haemor- rhage-a prospective case-control study. Thromb Res 2012; 129(5):e229-e232

25 Molyneux A, Kerr R, Stratton I, et al; International Subarachnoid Aneurysm Trial (ISAT) Collaborative Group. International Subarachnoid Aneurysm Trial (ISAT) of neurosurgical clipping versus endovascular coiling in 2143 patients with ruptured intracranial aneurysms: a randomized trial. J Stroke Cerebrovasc Dis 2002; 11(6):304-314

26 Kitsuta Y, Suzuki N, Sugiyama M, Yamamoto I. Changes in level of consciousness and association with hyperglycemia as tool for predicting and preventing re-bleeding after spontaneous subarachnoid hemorrhage. Prehosp Disaster Med 2006;21(3): 190-195

27 Sakai N, Yamada H, Ando T, Nishimura Y. Prevention of rebleeding after operation for subarachnoid hemorrhage of unknown cause. Neurosurgery 1985;17(6):942-946

28 Sakaki T, Morimoto T, Hoshida T, Kawaguchi S, Nakase H, Fukuzumi A. Rebleeding during transport of patients with a ruptured intracranial aneurysm. J Stroke Cerebrovasc Dis 1999;8(1):38-41

29 Kassell NF, Torner JC. Aneurysmal rebleeding: a preliminary report from the Cooperative Aneurysm Study. Neurosurgery 1983;13(5): 479-481

30 Cha KC, Kim JH, Kang HI, Moon BG, Lee SJ, Kim JS. Aneurysmal rebleeding : factors associated with clinical outcome in the rebleeding patients. J Korean Neurosurg Soc 2010;47(2):119-123

31 Tsuang FY, Su IC, Chen JY, et al. Hyperacute cerebral aneurysm rerupture during CT angiography. J Neurosurg 2012;116(6): $1244-1250$

32 Wu TC, Tsui YK, Chen TY, Lin CJ, Wu TC, Tzeng WS. Rebleeding of aneurysmal subarachnoid hemorrhage in computed tomography angiography: risk factor, rebleeding pattern, and outcome analysis. J Comput Assist Tomogr 2012;36(1):103-108

33 Molyneux A, Kerr R, Stratton I, et al; International Subarachnoid Aneurysm Trial (ISAT) Collaborative Group. International Subarachnoid Aneurysm Trial (ISAT) of neurosurgical clipping versus endovascular coiling in 2143 patients with ruptured intracranial aneurysms: a randomised trial. Lancet 2002;360(9342): 1267-1274

34 Komiyama M, Tamura K, Nagata Y, Fu Y, Yagura H, Yasui T. Aneurysmal rupture during angiography. Neurosurgery 1993; 33(5):798-803

35 Aoyagi N, Hayakawa I. Study on early re-rupture of intracranial aneurysms. Acta Neurochir (Wien) 1996;138(1):12-18

36 Tanno Y, Homma M, Oinuma M, Kodama N, Ymamoto T. Rebleeding from ruptured intracranial aneurysms in North Eastern Province of Japan. A cooperative study. J Neurol Sci 2007;258(1-2): $11-16$

37 Larsen CC, Astrup J. Rebleeding after aneurysmal subarachnoid hemorrhage: a literature review. World Neurosurg 2013;79(2): 307-312

38 Hellingman CA, van den Bergh WM, Beijer IS, et al. Risk of rebleeding after treatment of acute hydrocephalus in patients with aneurysmal subarachnoid hemorrhage. Stroke 2007;38(1): 96-99

39 Jamous MA, Nagahiro S, Kitazato KT, Tamura T, Kuwayama K, Satoh $K$. Role of estrogen deficiency in the formation and progression of cerebral aneurysms. Part II: experimental study of the effects of hormone replacement therapy in rats. J Neurosurg 2005;103(6): 1052-1057

40 Ruigrok YM, Slooter AJ, Rinkel GJ, et al. Genes influencing coagulation and the risk of aneurysmal subarachnoid hemorrhage, and subsequent complications of secondary cerebral ischemia and rebleeding. Acta neurochirurgica 2010;152(2):257-262 